\title{
Thermal and structural properties of spray pyrolysed CdS thin film
}

\author{
P RAJI, C SANJEEVIRAJA ${ }^{\dagger}$ and K RAMACHANDRAN* \\ School of Physics, Madurai Kamaraj University, Madurai 625 021, India \\ ${ }^{\dagger}$ Department of Physics, Alagappa University, Karaikudi 630 003, India
}

MS received 23 April 2004; revised 17 February 2005

\begin{abstract}
Using photo acoustic technique, the thermal properties of CdS thin films grown by spray pyrolysis are measured. Thermal diffusivity and conductivity in these films decrease at least two orders compared with bulk. These results are compared with our study on nano CdS and the other available literature. The comparison is good. The dependence of thermal diffusivity on the thickness of the layer or the size of the particles on the glass substrate are analysed from the present measurement and discussed. The dependence of thermal diffusivity on the thickness of the layer on the glass substrate is discussed.
\end{abstract}

Keywords. Spray pyrolysis; thin film; cadmium compounds; thermal diffusivity; thermal conductivity.

\section{Introduction}

$\mathrm{CdS}$ is a technologically useful material, as many devices based on $\mathrm{CdS}$, including sensors have come up in the recent years. The thin film cadmium sulphide solar cell has for several years been considered to be a promising alternative to the more widely used silicon devices.

Recently, Behera et al (2000) reported Raman measurements on nano crystalline CdS where unusual red shift in Raman spectra is observed when the excitation energy is decreased. We synthesized nano $\mathrm{CdS}$ and made photoacoustic measurements to correlate the above results (Raji et al 2004). The thermal parameters observed from this measurement reveal that there is atleast two orders of difference compared to the crystalline $\mathrm{CdS}(c-\mathrm{CdS})$. The particle sizes were also varied and we studied the band gap and thermal parameters. In all the cases, there is a marked difference between $c$-CdS and nano $\mathrm{CdS}$. The optical properties observed from photoacoustics reveal an usual trend of blue shifting when the energy is decreased.

In order to study further on the nano systems, due to varying reports on $\mathrm{CdS}$, these results are to be correlated and therefore, $\mathrm{CdS}$ thin films are prepared with the particle size in $\mathrm{nm}$ range.

Cadmium sulphide (CdS) can be deposited by various techniques like co-evaporation (Burton and Hench 1976) or elements evaporation (Romes et al 1976) from a concentric cylindrical source (Burton et al 1978). Afifi et al (1986) prepared evaporated thin film on glass substrate. Fraserk and Cook (1974) prepared CdS by rf diode sputtering technique. Padam et al (1988) have screen-printed $\mathrm{CdS}$ film on alumina substrates and sintered at $800^{\circ} \mathrm{C}$.

\footnotetext{
*Author for correspondence (thirumalchandran@yahoo.com)
}

One of the most promising techniques for producing large areas of inexpensive $\mathrm{CdS}$ film for terrestrial photovoltaic application is spray pyrolysis and here we followed this method to synthesize the CdS film. The structural properties of these films are carried out by X-ray diffraction technique and the optical study in the UV-VIS-NIR region, by spectrophotometer.

It is known that the measurement of thermal conductivity in thin films is not very simple and many times the figure of merit for the thin film could not be given because of this. Here, we report our measurements on the thermal properties of polycrystalline $\mathrm{CdS}$ thin film deposited on glass using photoacoustics and the two-layer model. Since the thin film is deposited on a glass substrate, any measurement on this will only be an effective value of both the film and the substrate.

In the case of two layer systems, experimental studies have been carried out for the effective thermal diffusivity and conductivity in terms of the thermal diffusivities and conductivities of the constituent layers (Mansanares et al 1990). In general, for studying these systems a thermal diffusion analogy model which was originally used in polymers and glasses, is used. However, this model has only been applied to systems like polymers and glasses.

\section{CdS film preparation}

The CdS thin films were prepared by taking a $0.5 \mathrm{M}$ aqueous solution of thiourea and $0.5 \mathrm{M}$ aqueous solution of cadmium chloride as starting solutions. Also CdS thin films were prepared from different concentrations of stock solutions: $0 \cdot 125 \mathrm{M}, 0 \cdot 25 \mathrm{M}$ and $0.375 \mathrm{M}$ of cadmium chloride and thiourea aqueous solution. The films were prepared from solutions having different mole ratios: $1 \mathrm{C} 1 \mathrm{~S}, 1 \mathrm{C} 2 \mathrm{~S}$ and $2 \mathrm{C} 1 \mathrm{~S}$ (where $1 \mathrm{C} 1 \mathrm{~S}$ stands for one part 
of cadmium chloride and one part of thiourea). The solutions were mixed thoroughly and the final solution was sprayed onto heated substrates kept at temperature ranging from $300-500^{\circ} \mathrm{C}$. The substrates were microscopic plane glass slides of area $3.5 \times 2.5 \mathrm{~cm}$. The usual cleaning of the glass slides were first carried out. The substrate heater was a cylindrical furnace designed indigenously. Chromelalumel thermocouple fixed on a dummy substrate holder was used to monitor the temperature of the substrates. When the solution is sprayed the following reaction takes place at the surface of the heated substrate,

$$
\mathrm{CdCl}_{2}+\left(\mathrm{NH}_{2}\right)_{2} \mathrm{CS}+2 \mathrm{H}_{2} \mathrm{O} \rightarrow \mathrm{CdS}+2 \mathrm{NH}_{4} \mathrm{Cl}+\mathrm{CO}_{2} \text {. }
$$

This yields a uniform growth of CdS films on the substrates. Each time $3 \mathrm{cc}$ of thiourea solution was sprayed on a heated substrate in order to prevent excessive cooling of the substrate.

The major preparatory parameters in the spray pyrolytic process are substrate temperature, the concentration and the mole rates of starting solutions. In this present work, the films were grown at air temperature; the spray rate was $18 \mathrm{cc} / \mathrm{min}$ and the distance between the spray nozzle and the substrate was $30 \mathrm{~cm}$. After deposition, the films were fast cooled to room temperature. The thickness of the films grown were quite uniform and free of pinholes throughout the samples.

The deposited CdS films are characterized for structural, optical and photo acoustic properties under different growth conditions. These grown films are distinguished in table 1 .

\section{Structural properties}

Films are prepared at $400,420,375^{\circ} \mathrm{C}$ as substrate temperature and the concentration of the precursor aqueous solution as $0.125 \mathrm{M}, 0.25 \mathrm{M}$ and $0.375 \mathrm{M}$. Golden yellow coloured films were obtained and the X-ray diffraction (XRD) patterns were recorded first for the spray deposited films. As an example, X-ray pattern for the film prepared at $400^{\circ} \mathrm{C}$ as the substrate temperature, $0 \cdot 375 \mathrm{M}$ as cadmium chloride and $0.400 \mathrm{M}$ as thiourea, is given in figure 2 in the volume ratio of $1: 1$ and all other spray parameters fixed as indicated in the previous section. From the XRD, the films are found to be in the single phase of $\mathrm{CdS}$ and the identification of the peaks indicate that the film is polycrystalline. The observed values are compared with ASTM data as shown in table 2. All the peaks agree with ASTM data for hexagonal cadmium sulphide. The prominent peak is (101) plane and the grain size is estimated as $59 \mathrm{~nm}$ from FWHM of this peak.

\section{Optical properties}

Optical absorbance against the wavelength is then observed for CdS thin films in the wavelength range 200-900 nm with glass as the reference and is shown in figure 3 . The absorbance spectrum shows a sharp increase in absorption at wavelength near to the absorption edge of the threshold wavelength for onset of absorption, the energy corresponding to this determines the band gap of the semiconductor material. The CdS film shows absorption coefficient $(\alpha)$ of about $5 \times 10^{4} \mathrm{~cm}^{-1}$ near the absorption edge. This shows that the deposited semiconductor film is a direct band gap material (Sze 1969). For allowed transition $(\alpha)^{2}$ is plotted against energy $(\mathrm{E}=h \gamma)$ to yield a straight line for direct transitions as shown in figure 3 from which the band gap is found to be $2.41 \mathrm{eV}$.

\section{Photo acoustic measurements}

Photo acoustic (PA) spectroscopy has received considerable attention in the last decade, because it is useful for material characterization when spectroscopic methods fail. In particular, opaque, polycrystalline or non-crystalline, biological systems and surfaces can be very effectively studied by photo acoustics (Rosencwaig 1975). Since CdS is highly absorbing, the photoacoustics measurements will yield better information than the other spectroscopic techniques.

In the present experiment, $400 \mathrm{~W}$ Xe-lamp (Jobin Yvon) is used as the light source. The sample (film + substrate) is placed in the photoacoustics (PA) cell and the mike is placed very close to the sample on the glass slide. To get the modulated light, a mechanical chopper (Model number PAR 650) is used with the source. The PA signal from the sample is fed to a lock-in amplifier (Model Perkin Elmer $7225 \mathrm{DSP})$. The light is allowed to fall on the sample through a monochromator (Model Triax 180, Jobin Yvon) as shown in figure 1.

Alvarado-Gil et al (1995) measured the thermal diffusivity for CdTe thin films, deposited on glass substrates

Table 1a. CdS films grown under different conditions.

\begin{tabular}{lcc}
\hline Sample & Temperature & Different conditions (spray time) \\
\hline Sample 1 & $420^{\circ} \mathrm{C}$ & $15 \mathrm{~s}, 3$ times \\
Sample 2 & $420^{\circ} \mathrm{C}$ & $15 \mathrm{~s}, 5$ times \\
Sample 3 & $420^{\circ} \mathrm{C}$ & $8 \mathrm{~s}, 3$ times \\
Sample 4 & $420^{\circ} \mathrm{C}$ & $8 \mathrm{~s}, 4$ times \\
Sample 5 & $420^{\circ} \mathrm{C}$ & $8 \mathrm{~s}, 5$ times \\
Sample 6 & $400^{\circ} \mathrm{C}$ & $8 \mathrm{~s}, 5$ times \\
Sample 7 & $400^{\circ} \mathrm{C}$ & $8 \mathrm{~s}, 6$ times \\
\hline
\end{tabular}

Table 1b. CdS films grown under different conditions.

\begin{tabular}{lcl}
\hline Sample & $\begin{array}{c}\text { Temperature and } \\
\text { spray time }\end{array}$ & $\begin{array}{c}\text { Different conditions } \\
\text { (morality) }\end{array}$ \\
\hline Sample 8 & $375^{\circ} \mathrm{C}, 5$ times & $0 \cdot 125 \mathrm{M}$ \\
Sample 9 & $375^{\circ} \mathrm{C}, 5$ times & $0 \cdot 5 \mathrm{M}$ \\
Sample 10 & $375^{\circ} \mathrm{C}, 5$ times & $0 \cdot 375 \mathrm{M}$ \\
\hline
\end{tabular}


by photoacoustic technique. Polycrystalline CdTe films having $55 \mu \mathrm{m}$ thickness were grown onto the glass slides using the close space vapour technique. The total thickness of two-layer system (glass and CdSe thin film) could be changed by varying the thickness of the thin film. They fitted their experimental data with a thermal diffusion analogy model. We follow their model here to fit our experimental data of $\mathrm{CdS}$ thin films.

$$
\alpha=\frac{1}{\frac{x^{2}}{\alpha_{1}}+\frac{(1-x)^{2}}{\alpha_{2}}+x(1-x)\left[\frac{\lambda}{\alpha_{1}}+\frac{1}{\lambda \alpha_{2}}\right]},
$$

where $x=l_{1} /\left(l_{1}+l_{2}\right), \lambda=\kappa_{1} / \kappa_{2}$.

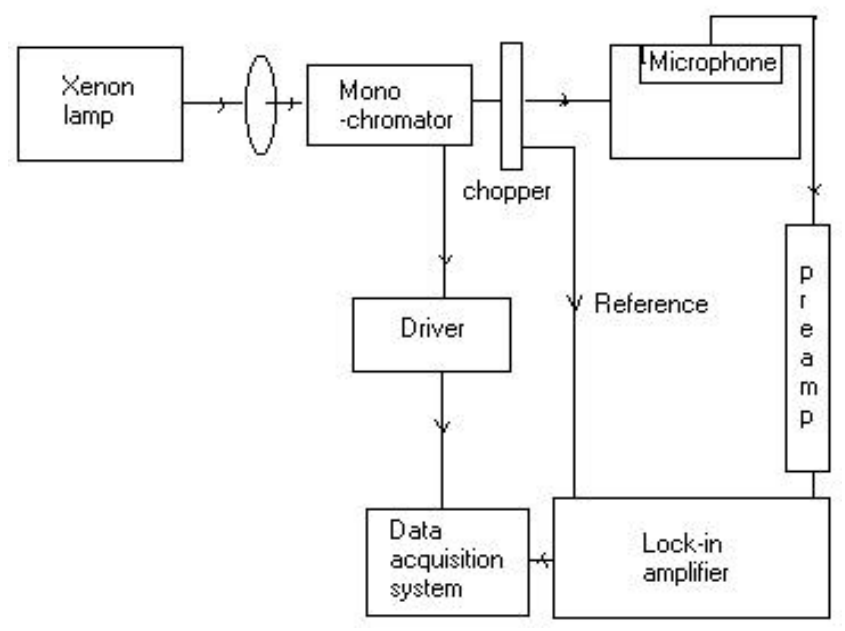

Figure 1. Photoacoustic experimental set-up.

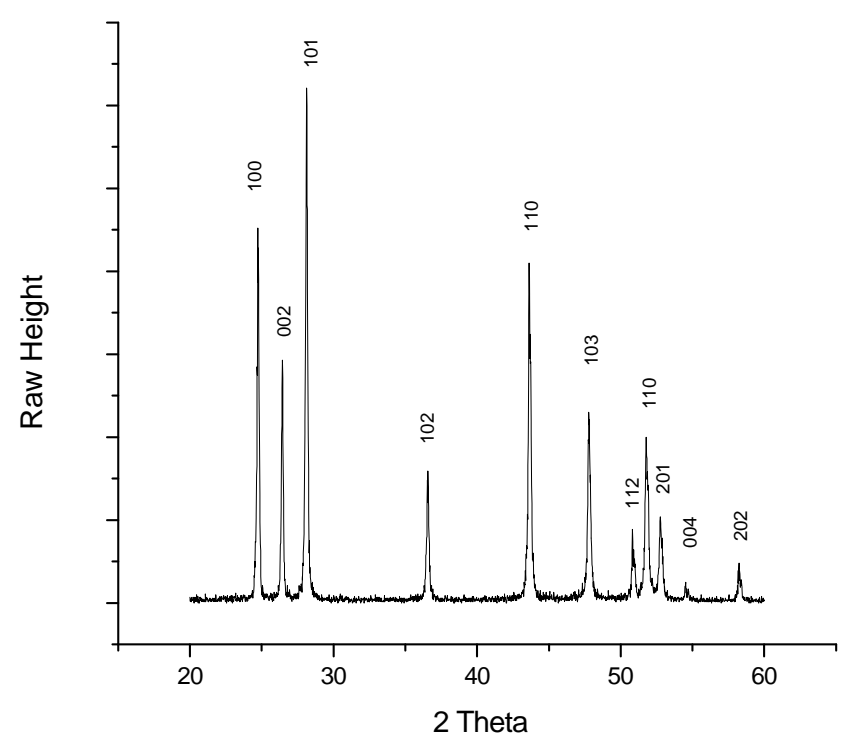

Figure 2. X-ray diffraction pattern for sample 6 .
Tominaga and Ito (1988) have shown the effective thermal diffusivity as

$$
\frac{l}{\sqrt{\alpha}}=\frac{l_{1}}{\sqrt{\alpha_{1}}}+\frac{l_{2}}{\sqrt{\alpha_{2}}},
$$

where $l_{1}, l_{2}$ are the thickness of glass and sample and $\alpha_{1}$ and $\alpha_{2}$ are their thermal diffusivities, respectively.

Similarly, the effective thermal conductivity is given as

$$
\frac{l}{\kappa}=\frac{l_{1}}{\kappa_{1}}+\frac{l_{2}}{\kappa_{2}},
$$

where $\kappa_{1}$ and $\kappa_{2}$ are thermal conductivities of glass and sample, respectively.

With this, following Rosencwaig and Gersho (1975) theory, the phase of the signal with respect to the reference $\operatorname{signal}(\Delta \varphi)$ is given as

$$
\tan (\Delta \varphi)=\tan h\left(l a_{\mathrm{s}}\right) \tan \left(l a_{\mathrm{s}}\right),
$$

where $l$ is the sample thickness and

$$
a_{\mathrm{s}}=\left|\frac{\pi f}{\alpha}\right|^{1 / 2},
$$

the thermal diffusion coefficient of the sample. The experimental measurements are done as follows.

The depth profile analysis by PA is carried out for two cases: (i) glass plate deposited with the film and (ii) glass plate alone (without the film).

Firstly the glass plate with thin film deposited is taken inside the PA cell and the PA signal is observed for different chopping frequencies. This is given in figure 4 for just one sample as an example. Thermal diffusivity is then calculated from the characteristic frequency of the sample. (The characteristic frequency is defined as the frequency at which the sample goes from thermally thick region to thermally thin region). This is found out from figure 5 where there is an $1 / f$ dependence on the PA signal.

If $f_{\mathrm{c}}$ is the characteristic frequency of the sample of thickness, $l$, then the thermal diffusivity can be calculated

\begin{tabular}{|c|c|c|c|c|}
\hline No. & $2 \theta$ & $d_{\mathrm{obs}}(\AA)$ & $d_{\mathrm{ASTM}}(\AA)$ & Plane \\
\hline 1 & $24 \cdot 746$ & $3 \cdot 5947$ & $3 \cdot 583$ & 100 \\
\hline 2 & $26 \cdot 435$ & $3 \cdot 3688$ & $3 \cdot 357$ & 002 \\
\hline 3 & $28 \cdot 137$ & $3 \cdot 1687$ & $3 \cdot 160$ & 101 \\
\hline 4 & $36 \cdot 570$ & $2 \cdot 4550$ & $2 \cdot 450$ & 102 \\
\hline 5 & 43.645 & $2 \cdot 0720$ & $2 \cdot 068$ & 110 \\
\hline 6 & $47 \cdot 793$ & $1 \cdot 9015$ & $1 \cdot 898$ & 103 \\
\hline 7 & $50 \cdot 826$ & 1.7949 & 1.790 & 200 \\
\hline 8 & 51.781 & $1 \cdot 7640$ & 1.761 & 112 \\
\hline 9 & $52 \cdot 767$ & 1.7333 & 1.731 & 201 \\
\hline 10 & $54 \cdot 525$ & $1 \cdot 6815$ & 1.682 & 004 \\
\hline 11 & $58 \cdot 249$ & $1 \cdot 5826$ & $1 \cdot 581$ & 202 \\
\hline
\end{tabular}
from

$$
\alpha=f_{\mathrm{c}} l^{2} \quad \mathrm{~m}^{2} \mathrm{~s}^{-1} .
$$

Table 2. Lattice spacing $(d)$ values for sample 6. 
Thermal conductivity is

$$
\kappa=\alpha \rho C_{\mathrm{p}} \quad \mathrm{Wm}^{-1} \mathrm{~K}^{-1},
$$

where $\rho$ is the density and $C_{\mathrm{p}}$ the specific heat capacity of the sample. Since the thin film deposited on the glass substrate is used as the sample, the thermal diffusivity measured here will be the effective thermal diffusivity, $\alpha$ (glass + film).

Such measurements are done for other CdS thin films also. Now for the glass plate alone as the sample, the PA signal was observed and with these two sets of data on thermal diffusivity of the glass plate $\left(\alpha_{1}\right)$ and the effective thermal diffusivity of glass plate and the film $(\alpha)$, thermal diffusivity for the film alone $\left(\alpha_{2}\right)$ is computed from (1). The measured thermal diffusivity of the glass here is $4.9 \times 10^{-7} \mathrm{~m}^{2} / \mathrm{s}$ which compares well with the litera-

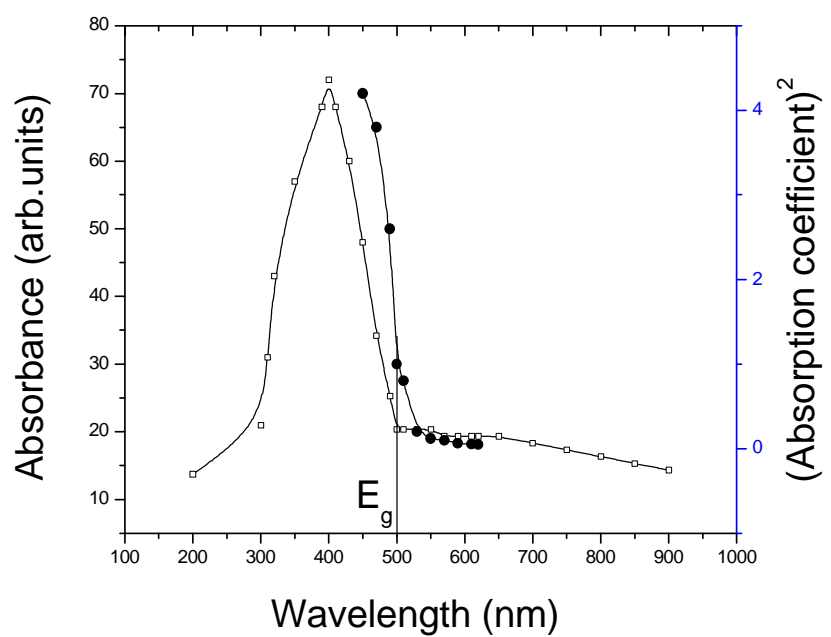

Figure 3. Optical absorbance curve and variation of $\alpha^{2}$ for sample 6 .

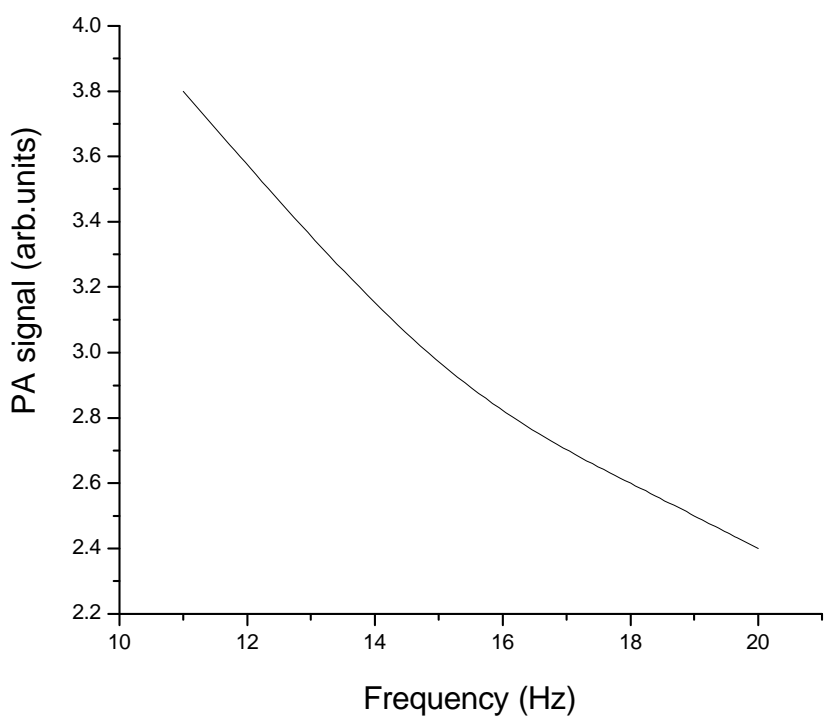

Figure 4. Depth profile analysis for sample 1. ture. The diffusivities of various CdS thin films thus measured are given in table 3 .

\section{Results and discussion}

Thermal properties of CdS films for different growth conditions by photoacoustics are very sparse in literature and here we report such measurements on these films by this technique. Even though few techniques are available for the thermal properties of thin films the reliability and uncertainty in these techniques have not been reported [http://www.vamas.org/twQ23.html]. Similarly, there is no consensus regarding the relationship between the thermal conductivity of thin film and bulk system.

Recently, Shen and Toyoda (2003) measured the thermal diffusivity of porous silicon by photoacoustics, where this porous silicon was deposited on a silicon substrate. When photoacoustic measurements were done, the value of thermal diffusivity of the substrate and the sample together were obtained. Then they used the two-layer model to find out the thermal diffusivity of porous silicon. This also supports the present use of two-layer model for the measurement of thermal diffusivity of CdS thin films.

Our measurements here on the CdS films (for different growth conditions) reveal a systematic trend. i.e. as the spray time increases, thermal conductivity decreases i.e. when thickness of the film is increased, more layers are deposited but the thermal diffusion length cannot be altered and so further thermal diffusion is not possible, after this thermal diffusion length.

For example, samples 1 and 3, 2 and 5 show this trend. Similarly, when the spraying trials are increased, there is uniform decrease in thermal diffusivity. For example, samples 3, 4 and 5 are prepared at the same conditions except the number of times the spraying done on the substrate are different. When more layers are deposited, there is again a decrease in thermal diffusivity. But when the substrate temperatures are decreased, as in samples 5 and 6 , there is an increase in the thermal diffusivity. When the temperature increases, it is known that additional defects and more porosity are likely to appear in the sample and due to these, phonon scattering will be higher and so there will be a decrease in thermal diffusivity. Gonzalez et al (2000) who studied thermal transport properties in $\mathrm{Al} / \mathrm{Ti}$ thin films, reported that the high defect density or the low mass density (due to porosity) would decrease thermal properties further. This has been comprehensively examined in review articles by Swartz and Pohl (1989) and Cahill (1997). It is known that when the temperature is increased, phonon scattering will be higher and consequently the mean free path will decrease and hence there will be a decrease in thermal diffusivity and thermal conductivity. On the other hand, when the temperature of the substrate is decreased, phonon mean free path increases and so the thermal conductivity. This is seen in samples 5 and 6. 
Table 3. Thermal parameters for various CdS films grown under different conditions.

\begin{tabular}{lccc}
\hline Sample type & $\begin{array}{c}\text { Thermal diffusivity } \\
\left(\times 10^{-9}\left(\mathrm{~m}^{2} / \mathrm{s}\right)\right)\end{array}$ & $\begin{array}{c}\text { Thermal conductivity } \\
\left(\times 10^{-3}(\mathrm{~W} / \mathrm{m}-\mathrm{K})\right)\end{array}$ & $\begin{array}{c}D \times 10^{-7}\left(\mathrm{~m}^{2} / \mathrm{s}\right) \text { for bulk } \\
\mathrm{CdS}(\mathrm{Ref})\end{array}$ \\
\hline Sample 1 & 3.62 & 4.81 & \\
Sample 2 & 3.56 & 4.74 & \\
Sample 3 & 3.83 & 4.93 & 3.5 \\
Sample 4 & 3.70 & $5 \cdot 1$ & 2000) \\
Sample 5 & 3.4 & 4.52 & \\
Sample 6 & 3.49 & 4.64 & \\
Sample 7 & 3.36 & 4.46 & \\
Sample 8 & 3.28 & 4.37 & \\
Sample 9 & 3.3 & 4.39 & \\
Sample 10 & 3.58 & 4.76 & \\
\hline
\end{tabular}

Error in measurements, $\sim 2 \%$.

Table 4. Effective thermal diffusivity values of glass-polycrystalline CdTe (Alvarado-Gil et al 1995).

\begin{tabular}{ll}
\hline$x=l_{1} /\left(l_{1}+l_{2}\right)$ & $\alpha\left(\mathrm{cm}^{2} / \mathrm{s}\right)$ \\
\hline 0 & 0.055 \\
$0 \cdot 31$ & 0.011 \\
0.69 & 0.0059 \\
0.76 & 0.0055 \\
0.79 & 0.0047 \\
0.833 & 0.0044 \\
1 & 0.0033 \\
\hline
\end{tabular}

Table 5. Effective thermal diffusivity values for CdS thin films.

\begin{tabular}{lcc}
\hline & $\begin{array}{c}\text { Effective thermal } \\
\text { diffusivity } \times 10^{-7} \\
\left(\mathrm{~m}^{2} / \mathrm{s}\right)\end{array}$ \\
Sample & $6 \cdot 21$ \\
Sample & 1 & $6 \cdot 04$ \\
Sample & 2 & $6 \cdot 43$ \\
Sample & 3 & $6 \cdot 62$ \\
Sample & 4 & $5 \cdot 78$ \\
Sample & 5 & $5 \cdot 92$ \\
Sample & 6 & $5 \cdot 54$ \\
Sample & 7 & $5 \cdot 32$ \\
Sample & 8 & 5.44 \\
Sample & 9 & $5 \cdot 84$ \\
Sample 10 &
\end{tabular}

All these values are then compared with bulk. It is found that the thermal diffusivity of these thin films (where the size of the particles are worked out from XRD, as few $\mathrm{nm}$ ) decrease atleast by two orders compared to the bulk.

Alvarado-Gil et al (1995) studied thermal diffusivity of CdTe on glass, for a series of different thicknesses of glass. The thermal diffusivity of the composite sample depends not only on the thermal diffusivity of its constituent materials but also on the ratio of their thermal conductivities. The thermal diffusivity of each constituent was measured separately, using PA phase-lag method by Alvarado Gil et al. Then the thermal conductivities were measured using the temperature rise method under conditions of white light illumination. Photoacoustic measurements on thin films depend solely on the thermal diffusion length of the sample and when the sample thickness is less than the thermal diffusion length of the sample then, only the substrate will play a role, as there will be reflection and transmission at the interface of the film and the substrate.

Eventhough our measurements are for multilayers (thickness of the film becoming larger and larger), the contributions from substrate is properly taken care by following the two-layer model of Alvarado Gil et al. The effective thermal diffusivity for the glass plate-polycrystalline CdTe, was calculated. Thus their measurements on the effective thermal diffusivity values increases by one order of magnitude, when CdTe is grown on the glass and the values are given in table 4.

The results reported here are only for $\mathrm{CdS}$ films and not the effective value of the film and the substrate. Still, we deposited CdS film on a quartz substrate (similar to sample 6) for verification. The film thickness is deliberately chosen as higher than the thermal diffusion length of $\mathrm{CdS}$ film. The result is same for thermal diffusivity for the film, as the two-layer model will take care of the substrate.

In our present study, we also find the same trend that there is a two-order decrease in thermal diffusivity of $\mathrm{CdS}$ thin film compared to crystalline $\mathrm{CdS}$. The size of the particles in both cases are few $\mathrm{nm}$. So, the quantum wavelength of lowest electronic state is constricted and consequently the minimum wavelength is shorter than the bulk solid. This explains the decrease in thermal properties. But when the thickness of the glass plate is fixed and thickness of the CdS is changed by the spray time, the effective thermal diffusivity increases two orders of magnitude than $\mathrm{CdS}$ when $\mathrm{CdS}$ is grown on the glass. The results are given in table 5 .

Hatta et al (2000) studied in detail the thermo physical properties of thin films on substrate by an a.c. calorimetric 
thermal diffusivity measurement. CVD diamond films made by Sumitomo Electric Co. (Itami, Japan) were used for the a.c. calorimetric measurement. There are three samples with thicknesses of $0.16,0.23$ and $0.53 \mathrm{~mm}$. The thicker samples were grown successively under the same condition and then, assumed the thickness of the 0th layer increases as the sample becomes thicker. In such a situation, the observed thermal diffusivities are fitted to the equation,

$$
D=D_{0}-\frac{E_{\mathrm{s}}}{d},
$$

where,

$$
\begin{aligned}
& D_{0}=\frac{\kappa_{0}}{c_{0}}, \\
& E_{\mathrm{s}}=\frac{\sum_{j=1}^{n}\left(\kappa_{0}-\kappa_{j}\right) d_{j}}{c_{0}},
\end{aligned}
$$

as $D_{0}$ and $E_{\mathrm{s}}$ are fitting parameters. Then, the following results are obtained

$$
\begin{aligned}
& D_{\mathrm{a}}\left[\mathrm{cm}^{2} / \mathrm{s}\right]=[12 \cdot 5 \pm 0.4]-\frac{[0 \cdot 48 \pm 0 \cdot 09]}{d[\mathrm{~mm}]}, \\
& D_{\mathrm{p}}\left[\mathrm{cm}^{2} / \mathrm{s}\right]=[12 \cdot 0 \pm 0.4]-\frac{[0 \cdot 37 \pm 0.08]}{d[\mathrm{~mm}]},
\end{aligned}
$$

where $D_{\mathrm{p}}$ and $D_{\mathrm{a}}$ are analysed from the data on the amplitude decay and the phase shift in the a.c. temperature, and $d$ the thickness of the layer and 12.5 is the thermal diffusivity for bulk, respectively. This clearly shows that there will be a drastic reduction in the value of thermal diffusivity by two to three orders (as the thickness of the layer will be in microns).

So, in our present work, the two-layer model, which is found to be successful in thin films, when used, thickness dependence on thermal diffusivity for $\mathrm{CdS}$ thin film is in accordance with the previous results reported in the literature. Thus the photoacoustics could be used to measure the thermal conductivity of the thin films and hence the figure of merit.

\section{Conclusions}

Photoacoustic measurements on thin films for thermal diffusivity and thermal conductivity are more reliable than any other known methods because, here the sample is not touched by any of the probe while making measurements. Four-probe method, mirage techniques etc are destructive as thin films can be easily damaged by excessive heating and in such cases touching the films by probes is not advisable and such measurements are less reliable. But here in photoacoustics, these are avoided and so the results are reliable. The present measurements show that the thermal transport properties are small compared to the bulk CdS. This is in conformity with earlier measurements on various other films and in general it is recognized that thermal transport properties of thin films are less than half of those of bulk materials (Lee and Cahill 1997). Here accurate measurements are made on CdS thin films to show that it may be even two orders less compared to bulk CdS.

\section{References}

Afifi H A, Kenaway M A, El Nahass M M, ElZahid H L and Elshaly A A 1986 Indian J. Pure \& Appl. Phys. 20550

Alvarado-Gil J J, Zelaya-Angel $\mathrm{O}$ and Sanchez-Sinencio $\mathrm{F}$ 1995 Vac. 46883

Behera S N, Sahu S N and Nanda K K 2000 Indian J. Phys. A74 81

Burton L C and Hench T L 1976 Appl. Phys. Lett. 29612

Burton L C, Baron B, Hench T L and Meakin J D 1978 J. Elec. Mater. 7159

Cahill D G 1997 Microscale Thermophysical Engineering 1 85

Fraserk D B and Cook H D 1974 J. Vac. Sci. Technol. 1156

Gonzalez E J, Bonevich J E, Stafford G R, White G and Josell De 2000 J. Mater. Res. F71 855

Hatta Ichiro, Kazuhiko Fujii and Sok Won Kim 2000 Mater. Sci. Eng. A292 189

Hernadez-Guevara A, Cruz-orea A and Sanchez-Sinencio F 2000 Superficies Yvacio $\mathbf{1 0} 51$

Lee S M and Cahill D G 1997 J. Appl. Phys. 812590

Mansanares A M, Bento A C, Vargas H, Leite N F and Miranda L C M 1990 Phys. Rev. B42 4447

Padam G K, Shankar V and Ghosh P H 1988 J. Mater. Sci. 23 1064

Raji P, Sanjeeviraja C and Ramachandran K 2004 Cryst. Res. Technol. 39617

Romes N, Sbervegluri G and Tarricone L 1976 Appl. Phys. Lett. 328

Rosencwaig A 1975 Phys. Today 2823

Rosencwaig A and Gersho A 1975 J. Appl. Phys. 4764

Shen Qing and Toyoda Taro 2003 Rev. Sci. Instrum. 74601

Swartz E T and Pohl R O 1989 Rev. Mod. Phys. 61605

Sze S M 1969 Physics of semiconductor devices (New York: Wiley) 2 edn

Tominaga T and Ito A 1988 Space Appl. Phys. 272392 